Ovarium cum corolla basi coalitum, apice valde concavum, 10-loculare; ovulis in unoquo que loculo plurimis; stigmata 5, apice 2-3-fida, arcte conniventia, ad basin corollæ inserta.

Herba tota $17 \mathrm{~cm}$. alta. Foliorum petiolus $5-7 \mathrm{~cm}$. longus, lamina $10 \mathrm{~cm}$. longa, 20-25 mm. lata, auriculis 3-5 mm. longis. Pedunculus 5-6 cm. longus. Sepala $20 \mathrm{~mm}$., cum mucrone $25 \mathrm{~mm}$. longa. Corolla $15 \mathrm{~mm}$. alta.

Indo-Cuns. - Cochinchine : plante croissant au fond des ruisseaux des forêts, fleurit pendant la saison des pluies, $n^{\circ} 1381$ [Thorel].

Il existe actuellement 2 espèces de cet intéressant petit genre : le Barclaya longifolia Wall. qui se rencontre aux lles Andaman, en Birmanie et en Cochinchine et le $\boldsymbol{B}$. Mottleyi Hooker, originaire de Bornéo. La $3^{e}$ espèce est la présente qui conservera ici le nom que le $\mathrm{D}^{\mathrm{r}}$ Thorel lui a donné dans le répertoire général de son herbier. Elle a beaucoup d'analogie avec le $B$. longifolia Wall., mais elle s'en distingue : $1^{\circ}$ par les feuilles moitié plus courtes et étroites, à 9 paires de nervures au lieu de 11-12; $2^{\circ}$ par 4-5 pétales au lieu de $8-10 ; 3^{\circ}$ par 5 stigmates bifides, séparés par des sinus beaucoup plus profonds que leur bifidité.

M. F. Pelourde fait la communication suivante :

\title{
Recherches sur la position systématique des plantes fossiles dont les tiges ont été appelées
} Psaronius, Psaroniocaulon, Gaulopteris;

par m. Fernand pelourde.

On sait que l'alliance des Marattiales, qui est représentée aujourd'hui par une seule famille et un petit nombre de genres et d'espèces, avait pris, aux temps primaires, une extension considérable. A cette époque, les individus qui la constituaient présentaient dans leurs racines, dans leurs tiges et dans leurs organes fructificateurs une structure analogue à celle que l'on observe maintenant dans les plantes du même groupe ${ }^{1}$. Mais on admettait jusqu'ici qu'ils possédaient une organisation foliaire spéciale. Une série de recherches comparatives que j’ai faites entre les Marattiacées vivantes et fossiles m'a permis de démontrer le contraire : j'ai constaté en effet que, dans les unes

1. Voir notamment à ce sujet: Grand'EuRY (C.), Flore carbonifere du département de la Loire et du centre de la France (Mém. sav. étrangers Ac. sc., 1877). - Rudolph (KARL), Psaronien und Marattiaceen... (Denksch. d. kaiserlichen Akademie d. Wissensch., Bd. 78, Vienne, 1906.) 
F. pelourde. - psaronius, psaroniocaulon, caulopteris.

comme dans les autres, l'appareil conducteur des frondes est construit exactement sur le même plan ${ }^{1}$.

\section{Marattiacées vivantes.}

Marattia fraxinea. - J'étudierai d'abord un certain nombre de Marattiacées vivantes en commençant par le Marattia fraxinea Sm., par exemple. Dans cette espèce, on remarque, à la base du rachis principal des feuilles, une série externe de faisceaux ordonnés, en coupe transversale, suivant une ellipse (Pl. III, fig. 1). A l'intérieur de celle-ci, il existe un petit nombre d'autres faisceaux, figurant, du côté inférieur, un arc à courbure assez prononcée et, du côté supérieur, une bande presque rectiligne; l'ensemble ainsi constitué représente une deuxième ellipse à peu près concentrique à la première. A un niveau plus élevé, par suite de fusions entre ces divers cordons libéroligneux, la bande supérieure est réduite à un seul faisceau. $\mathrm{Si}$ l'on s'élève encore davantage, on constate que l'arc inférieur ne comprend plus également qu'un faisceau, qui est très allongé, et dont la forme est encore arquée (Pl. III, fig. 2). Durant cette transformation, le faisceau supérieur se rapproche de plus en plus du contour de l'ellipse externe, et, vers le lieu d'insertion de la première paire de pennes, il se fusionne avec un des faisceaux de cette ellipse. On n'a plus alors qu'un seul faisceau interne allongé et à peu près rectiligne. Ce faisceau se fusionne ultérieurement avec deux autres, appartenant à la partie supérieure de l'ellipse externe et présentant une concavité du côté opposé à celui qui regarde le plan de symétrie; pour cela, les deux faisceaux en question se soudent entre eux suivant une partie de leur surface convexe et ils se soudent aussi à la portion médiane du faisceau interne. On obtient ainsi un faisceau

1. Ce travail, dont j’ai déjà exposé brièvement les principaux résultats (C. R. Acad. Sc., 25 novembre 1907), a été fait au laboratoire de Cryptogamie du Muséum, sous la direction de mon maître, M. le professeur MANGIN, à qui j'exprime ma vive reconnaissance. J'assure également de ma profonde gratitude M. ZEILLER, membre de l'Institut, pour les documents qu'il m'a communiqués et pour les conseils qu'il m'a donnés. J'adresse aussi mes remerciements à M. le professeur LECONTE, qui a bien voulu me laisser étudier les échantillons de paléobotanique du Muséum, ainsi qu'à MM. les assistants BONNET et HARIOT. 
ayant une forme d'X. La fig. III, Pl. 4, montre le commencement de ces unions.

Vers le niveau de la deuxième paire de pennes, le faisceau en $X$ se fractionne suivant un plan perpendiculaire au plan de symétrie du rachis et qui passe par sa partie médiane. On a alors, comme précédemment, une ellipse externe et un faisceau interne très allongé. On arrive ainsi à l'extrémité du rachis principal, qui est prolongé par la penne terminale. Dans le renflement qui est situé à la base de cette penne, le faisceau interne s'incurve en son milieu, de façon à devenir concave du côté inférieur; puis il se divise en deux autres (Pl. 1II, fig. 3), qui sont concaves du côté externe, et qui donnent à l'ensemble du système fasciculaire la forme d'un arc ouvert en haut et dont les bords sont recourbés en dedans. Le nombre des faisceaux qui constituent cet arc diminue de plus en plus et, à un certain moment, on n'en a plus que trois principaux : un inférieur et deux supérieurs, lesquels arrivent à se souder bout à bout. On a ainsi en tout deux faisceaux, qui, au niveau de l'avant-dernière pinnule, se coupent chacun en deux autres; puis, une moitié de chacun d'eux se dirige vers la pinnule, et ces deux moitiés se soudent par leurs extrémités, de façon à former un faisceau cylindrique, dont la trace est représentée par une couronne, en coupe transversale. Ensuite ce faisceau se fend du côté supérieur et acquiert la forme d'un arc concave en haut et dont les bords sont recourbés vers l'intérieur. Quant aux deux autres moitiés, elles se comportent de la mème façon dans l'extrémité du rachis principal et dans la pinnule qui le prolonge; cette pinnule terminale et l'extrémité du rachis constituent donc un ensemble équivalant à l'avant-dernière pinnule, au point de vue de l'appareil conducteur : c'est là un exemple de véritable dichotomie ${ }^{1}$ (Pl. III, fig. 5).

Si l'on considère maintenant une penne appartenant à la première paire, on y remarque, à la base, trois faisceaux : un supérieur, un inférieur et un intermédiaire; après un certain nombre de divisions, on observe un cercle externe de faisceaux,

1. Ce n'est pas là un cas isolé chez les Fougères. Thome a observé des faits du même ordre dans la fronde de l'Aspidium dilatatum et dans celle du Marattia arguta (Die Blattstiele der Farne, p. 121; pl. V, fig. 12, et pl. VI, fig. 16). 
F. pelourde. - psaronius, psaroniocaulon, Caulopteris.

et un faisceau interne, comme dans le rachis principal. Le faisceau interne s'unit par chacune de ses extrémités à un des faisceaux du cercle externe; sa partie ligneuse se coupe en deux, et chaque moitié va se fusionner avec le bois d'un des deux faisceaux en question. Puis, il se fend complètement en deux parties, et l'on obtient, au lieu des trois faisceaux qui viennent de s'unir, deux faisceaux arqués, à concavité tournée du côté externe. L'ensemble présente alors une forme d'arc analogue à celui du rachis principal. A partir de l'avant-dernière pinnule, les choses se passent comme dans ce dernier.

Dans la deuxième paire de pennes, le système fasciculaire subit des transformations analogues à celles quill subit dans la première paire.

Ainsi, dans la feuille du Marattia fraxinea, il existe, au point de vue de la distribution des faisceaux, deux types principaux: le premier est caractérisé par un contour externe de faisceaux, $\dot{a}$ l'interieur duquel se trouvent un ou plusieurs autres faisceaux qui, à partir d'un certain niveau, sont ordonnés suivant une bande transversale, arquée ou rectiligne; le second est caractérisé par un arc vasculaire ouvert du côté supérieur, à bords recourbés en dedans et qui devient continu dans les pinnules.

Marattia Kaulfussii. - Dans le Marattia Kaulfussii J. Sm., on remarque, à la base du rachis principal des frondes, un certain nombre de faisceaux ordonnés suivant un contour fermé, triangulaire ou elliptique, en tous cas aplati du côté supérieur, et à l'intérieur duquel se trouve un autre faisceau ayant la forme d'une bande aplatie (PI. III, fig. 6). Ce dernier faisceau ne tarde pas à se diviser en deux autres, convexes du côté du plan de symétrie et donnant à l'ensemble une forme générale d'arc (PI. III, fig. 7), comme dans le $M$. fraxinea. Puis, les deux faisceaux internes se rapprochent l'un de l'autre et se soudent bout à bout, de façon à n'en plus former qu'un seul, lequel se coupe ensuite en trois autres, au niveau de la première paire de pennes (Pl. III, fig. 8). Peu après, il ne reste plus de ceux-ci que le faisceau médian, lequel se coupe en deux autres, de façon à redonner encore à l'ensemble une forme d'arc. Cette forme se maintient jusqu'au sommet du rachis principal. A un certain moment, l'arc ne se compose plus que de trois fais- 
ceaux, un inférieur et deux supérieurs, el, au-dessus de la cinquième paire de pennes, il est constitué par un seul faisceau continu.

Toutes les ramifications du rachis principal possèdent un appareil conducteur disposé en arc et constitué, soit par un très petit nombre de faisceaux, soit par un seul faisceau, suivant la taille et le niveau des parties que l'on examine.

Ainsi, l'étude de la fronde du Marattia Kaulfussii montre combien les deux types de structure que j'ai déjà signalés dans le $M$. fraxinea peuvent se transformer facilement l'un dans l'autre : une simple fragmentation du faisceau interne, ou une réunion des faisceaux ainsi obtenus suffisent pour opérer cette transformation.

Angiopteris evecta. - Si l'on examine ensuite une penne d'Angiopteris evecta Hoffm., on remarque, à la base de son rachis, deux séries de faisceaux ordonnés à peu près comme à la base du pétiole, chez le $M$. fraxinea (PI. III, fig. 9). Ceux de la série interne sont rangés, du côté inférieur, sur deux lignes formant entre elles un angle obtus ouvert en haut, et, du côté supérieur, ils forment un arc dont la concavité regarde l'ouverture de l'angle précédent. Un des faisceaux de cet arc se rapproche de l'un de ceux de la série externe et se fusionne avec lui. On n'a plus alors, à l'intérieur de la série externe, qu'un arc à concavité tournée du côté supérieur, au lieu d'une ligne fermée (Pl. III, fig. 10). Cet arc lui-même se transforme peu à peu en une bande à peu près rectiligne, perpendiculaire au plan de symétrie et composée de quelques larges faisceaux aplatis, dont l'un est beaucoup plus important que les autres (Pl. III, fig. 11). Ce dernier émet sur sa face supérieure un prolongement qui arrive à fusionner avec un des faisceaux de la série externe. Le faisceau résultant de cette union a une forme d'X (Pl. III, fig. 12). Puis, il se divise dans le sens du plan de symétrie du rachis et se transforme en deux autres, concaves du côté externe. L'ensemble de tous les faisceaux présente alors la forme d'un arc ouvert en haut et dont les extrémités sont recourbées en dedans (Pl. III, fig. 13). Puis, les deux faisceaux provenant de la division précédente se réunissent en un nouvel $\mathbf{X}$, lequel se divise ensuite suivant un plan perpendiculaire au 
F. pelourde. - pSaronius, psaroniocaulon, caulopteris.

plan de symétrie. On obtient donc à nouveau une ligne externe de faisceaux fermée et, en dedans de celle-ci, une bande presque rectiligne. Puis, il se reforme un $X$, comme précédemment, et, par division de cet $X$, un arc semblable à celui que j'ai décrit tout à l'heure. Vers le sommet du rachis, cet arc ne comprend plus que trois faisceaux : un inférieur et deux supérieurs. Les deux supérieurs se réunissent ensuite par une de leurs extrémités, et l'on n'a plus que deux faisceaux en tout. Le système libéro-ligneux ainsi constitué se répartit d'une façon à peu près égale entre l'avant-dernière pinnule et le reste du rachis.

Les deux dernières pinnules, ainsi que toutes les autres folioles de la fronde, renferment deux faisceaux à leur base. Leur faisceau supérieur se coupe en deux autres, qui se soudent chacun à une des extrémités du faisceau inférieur, tantôt l'un après l'autre, tantôt simultanément. Ou bien, les deux faisceaux primitifs se réunissent par leurs extrémités avant que le supérieur ne se soit divisé. En tout cas, on obtient finalement, dans la nervure médiane de chaque foliole un faisceau unique, en forme d'arc ouvert en haut, et à extrémités recourbées en dedans (Pl. III, fig. 14 et 15). Cet arc est semblable à ceux que l'on a déjà observés dans le rachis principal, sauf qu'il est continu au lieu d'être dissocié.

Angiopteris d'Urvilleana. - On peut encore retrouver des phénomènes du même ordre dans une penne d'Angiopteris d'Urvilleana de Vriese. A la base de cette penne, on rencontre une ligne externe de faisceaux fermée, à peu près circulaire et, à son intérieur, deux larges faisceaux arqués, l'un supérieur, l'autre inférieur, figurant à eux deux un second cercle excentrique par rapport au premier (PI. III, fig. 16).

Le supérieur se rapproche d'un des faisceaux du cercle externe et se soude à lui par une de ses extrémités (Pl. IV, fig. 17). Au bout d'un certain temps, il ne reste plus à l'intérieur du cercle externe que le faisceau supérieur primitif, qui est maintenant aplati au lieu de présenter la forme de gouttière qu'il avait tout à l'heure (Pl. IV, fig. 18). Puis, ce faisceau interne s'unit à deux autres appartenant à la partie supérieure du cercle externe, de façon à donner un faisceau en $X$, comme 
chez les espèces précédentes. Cet $X$ se partage, suivant le plan de symétrie du rachis, en deux faisceaux concaves du côté externe et donnant à l'ensemble la forme d'arc habituelle. Quelque temps après, on obtient de nouveau un $\mathbf{X}$ semblable au premier et qui se redivise presqu'aussitôt comme précédemment. Dans le reste du rachis, ainsi que dans les diverses pinnules, les choses se passent comme chez l'Angiopteris evecta.

L'étude d'un certain nombre d'autres espèces, provenant de l'herbier du Muséum, m'a permis de constater que, dans tous les genres de Marattiacées vivantes, on retrouve toujours, dans l'appareil conducteur des feuilles, les deux types d'organisation dont j'ai parlé plus haut.

Kaulfussia. - C'est ainsi que, dans le Kaulfussia æsculifolia $\mathbf{B l}$. par exemple, j'ai observé, dans un morceau de pétiole, une série externe de faisceaux ayant, en coupe transversale, la forme d'un triangle isocèle, dont la base est tournée du côté supérieur; à l'intérieur de ce triangle, j'ai remarqué deux faisceaux ordonnés suivant une bande transversale.

J'ai examiné également la base d'une foliole et, dans sa nervure médiane, j'ai vu d'abord un certain nombre de faisceaux rangés suivant un contour elliptique à l'intérieur duquel se trouvent deux autres faisceaux. Ceux-ci se fusionnent bientôt avec quelques autres, appartenant à la série externe, de façon à donner un faisceau en $\mathbf{X}$. Ce dernier se divise en deux parties suivant le plan de symétrie de la nervure (Pl. IV, fig. 19), et on obtient ainsi, dans l'ensemble, la forme d'arc habituelle (Pl. IV, fig. 20). Dans une nervure latérale, j'ai constaté la présence d'un seul faisceau arqué continu, à concavité assez peu prononcée et tournée du côté supérieur (Pl. IV, fig. 21).

Danæa. - Dans les Danæa alata Sm. et elliptica Sm., ainsi que dans l'Angiopteris uncinata de Vriese et le Marattia sambucina $\mathrm{Bl}$., j’ai encore observé dans des fragments de pétioles une série externe de faisceaux fermée, avec, à son intérieur, une bande transversale constituée généralement par un seul faisceau; ce dernier est large et aplati, ou bien il présente la forme d'une gouttière à concavité tournée du côté supérieur.

Dans le Danza elliptica et dans le $D$, polyphylla, la nervure 
médiane des folioles m'a montré, à une certaine distance de la base de celles-ci, un appareil conducteur presque continu, ayant la forme d'un angle aigu dont le sommet est tourné du côté inférieur, et dont les bords libres sont recourbés vers l'intérieur (Pl. IV, fig. 22). Cet appareil, qui devient tout à fait continu à un certain niveau, est analogue à l'arc que j’ai signalé précédemment. Sa forme anguleuse est une simple modification de détail, en corrélation avec celle du pétiole.

J'ajouterai enfin que j'ai encore constaté dans la nervure médiane des folioles du Marattia cicutæfolia Kaulf., la présence d'un faisceau unique, ayant la forme d'arc ordinaire.

En résumé, d'après ce qui précède, on peut dire que la disposition des faisceaux dans les feuilles des Marattiacées se ramène

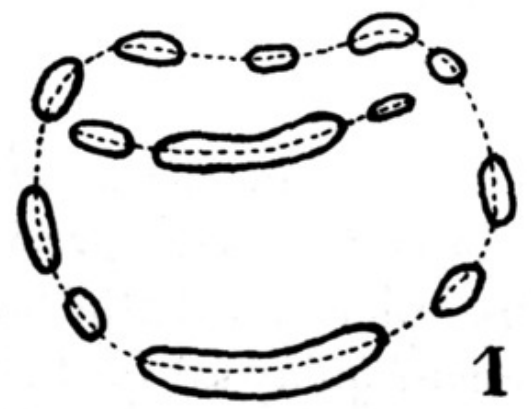

Fig. 1. - Coupe transversale schématique de l'appareil conducteur d'un rachis de Marattiacee vivante, montrant la ligne externe de faisceaux fermée, et la bande interne ( $1^{\mathrm{er}}$ type).

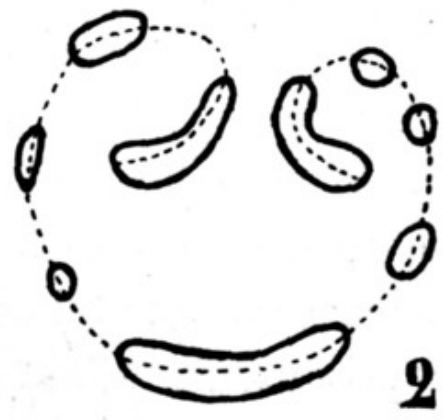

Fig. 2. - Coupe transversale schématique d'un rachis de Marattiacée vivante, montrant les faisceaux ordonnés suivant un arc ouvert du côté supérieur, et à bords recourbés en dedans ${ }^{\prime} 2$ type).

aux deux types fondamentaux suivants : tantôt on observe, sur les coupes transversales, un contour externe de faisceaux fermé, renfermant à son intérieur d'autres faisceaux, qui constituent une ligne arquée, concave en haut, ou une bande transversale à peu près rectiligne (fig. 1); tantôt l'ensemble des faisceaux est ordonné suivant un arc ou un $U$, ouvert du côté supérieur, et dont les bords se recourbent en crochets vers l'intérieur (fig. 2). Cet arc, d'abord dissocié, devient continu, c'est-à-dire constitué par un faisceau unique, dans les dernières ramifications des frondes et, en particulier, dans les pinnules.

On trouve tous les passages entre ces deux manières d'être, qui peuvent d'ailleurs se retrouver à plusieurs niveaux diffé- 
rents dans un même organe, comme dans les pennes de l'Angiopteris evecta, par exemple.

Ce plan d'organisation est tout à fait caractéristique de la famille des Marattiacées ${ }^{1}$; bien qu'il n'ait pas une très grande importance physiologique, il présente un grand intérêt au point de vue de son application à l'étude des Marattiacées fossiles, chez lesquelles nous allons le retrouver exactement.

(A suivre.)

M. Lutz, secrétaire général, lit la Note suivante de M. Russell :

\section{Observations sur des Genêts à balais adaptés à un sol calcaire;}

PAR M. W. RUSSELL.

Le Genêt à balais (Sarothamnus scoparius Koch) a, on le sait, une appétence très faible pour la chaux : aussi la présence de cet arbrisseau en dehors des terrains siliceux est-elle tout à fait exceptionnelle. C'est pour cette raison qu'ayant eu l'occasion de rencontrer une colonie de Genêts égarée sur un sol calcaire, il m'a paru intéressant de rechercher par quelle sorte d'artifice ces Genèts arrivaient à se maintenir dans un milieu qui leur est d'ordinaire des plus funestes.

Les plantes en question s'observent au sommet des coteaux de Lardy (Seine-et-Oise) sur une sorte de promontoire qui domine la route de Torfou; elles vivent dans des marnes sableuses contenant de nombreux graviers calcaires très friables et sont associées à des espèces calciphiles comme Ophrys aranifera Huds., Hippocrepis comosa L., Tussilago Farfara L., Inula Conyza DC., etc. L'indice calcimétrique moyen de la terre finement tamisée est de 6,833 , quantité considérable pour l'appétence

1. Voir notamment, sur l'anatomie des Marattiacées : De Vries et Harting, Monographie des Marattiacées..., Leyde et Dusseldorf, 1853, fig. 16, pl. VII. - KüHN, Untersuchungen über die Anatomie der Marattiaceen und anderer Gefässkryptogamen, Flora, 1889. - BREBNER, On the anatomy of Danæa and other Maraltiacex, Annals of Botany, XVI, 1902, fig. 11, 12, 14, 15, 17, pl. XXII. - Tноме, Die Blattstiele der Farne..., Jahrb. f. wissensch. Bot., t. XVII, 1886, p. 118-119, et fig. 16, 17, 18, pl. VI; etc. 


\section{$2 \mathrm{BHL}$ Biodiversity Heritage Library}

Pelourde, M Fernand. 1908. "Recherches sur la position systématique des plantes fossiles dont les tiges ont été appelées Psaronius, Psaroniocaulon, Caulopteris." Bulletin de la Société botanique de France 55, 88-96. https://doi.org/10.1080/00378941.1908.10831343.

View This Item Online: https://www.biodiversitylibrary.org/item/8677

DOI: https://doi.org/10.1080/00378941.1908.10831343

Permalink: https://www.biodiversitylibrary.org/partpdf/160687

\section{Holding Institution}

Missouri Botanical Garden, Peter H. Raven Library

\section{Sponsored by}

Missouri Botanical Garden

\section{Copyright \& Reuse}

Copyright Status: Public domain. The BHL considers that this work is no longer under copyright protection.

This document was created from content at the Biodiversity Heritage Library, the world's largest open access digital library for biodiversity literature and archives. Visit BHL at https://www.biodiversitylibrary.org. 